Anaesthesist 2015 • 64:811-813

DOI 10.1007/s00101-015-0112-9

Online publiziert: 9. November 2015

(c) Springer-Verlag Berlin Heidelberg 2015

\section{Rehm}

Klinik für Anaesthesiologie, Klinikum der Universität München, Campus Großhadern, München, Deutschland

\title{
Wissen-schafft Sicherheit!
}

Dieses Motto passt zu dem Leitthemenbeitrag von Boehm et al.: „Perioperatives Risiko und Letalität nach großen chirurgischen Eingriffen " in der vorliegenden Ausgabe von Der Anaesthesist, denn diese ausgesprochen lesenswerte Arbeit beinhaltet die sorgfältige wissenschaftliche Auseinandersetzung mit der Frage: Wie sicher ist Anästhesie/Narkose eigentlich?

Weltweit werden pro Jahr rund 230 Mio. Narkosen durchgeführt; in Deutschland sind es über 10 Mio. Trotzdem haben viele Patienten Angst vor einer Narkose. Angst, weil sie das Gefühl haben, die Kontrolle über ihre Vitalfunktionen zu verlieren, nach einer Narkose nicht wieder aufzuwachen oder während einer Narkose zu sterben. Zumindest die Angst vor dem Kontrollverlust über die Vitalfunktionen ist auf gewisse Weise paradox und damit unbegründet. Hierzu ein Beispiel: Sie als geneigter Leser können sich, während Sie sich gerade der Lektüre dieser Zeilen zuwenden, ganz in Muße auf die Inhalte dieses Textes konzentrieren, ohne deshalb die geringste Sorge haben zu müssen, einen Kontrollverlust hinsichtlich Ihrer Vitalfunktionen zu erleiden. Dies liegt daran, dass Sie, wie alle anderen Menschen natürlich auch, gar keine willkürliche und unbeschränkte Kontrolle über Ihre Vitalfunktionen besitzen. Niemand weiß, wann das eigene Herz womöglich einmal aussetzen wird, ein Ereignis, das leider so unwahrscheinlich gar nicht ist. Ein plötzlicher Herztod ereignet sich allein in Deutschland zwischen 100.000- und 200.000-mal pro Jahr. Man kann seinen Herzschlag nur äußerst eingeschränkt durch Willenskraft beeinflussen. (Dies gilt natürlich für den Normalmenschen, Apnoetaucher mögen hier eine Ausnahme bilden.) Auch die Atmung läuft weitestgehend unwillkürlich, sprich automatisch ab. Gott sei Dank, müssen wir das auch nicht willkür- lich kontrollieren, denn dies würde jeden Menschen unzweifelhaft überfordern, bedenkt man, dass dies lebenslang ohne Pause durchgehalten werden müsste. Der Mensch hat also eine Art „Automatik“ oder komplexen „Autopilot“ eingebaut, für den es - anders als im Cockpit eines Flugzeugs - keinen einfachen Ein- und Aus-Schalter gibt.

Gleichwohl, selbst wenn der Mensch bzw. der Patient selbst keine wirkliche Kontrolle über seine Vitalfunktionen hat und, so gesehen, diese dem Anästhesisten auch nicht wirklich „übergeben“"kann, ist der Eingriff durch den Anästhesiologen während einer Narkose für den Patienten in der Regel massiv.

$>$ Anästhesie beinhaltet häufig das Ausschalten des Bewusstseins, etwas durchaus „Existenzielles" für den Menschen.

Dies ist ein Vorgang, den der medizinische Laie oft nur schwer verstehen kann und der naturgemäß deshalb schon Angst erzeugt. Ursache ist, dass die Wirkungen von Anästhetika sehr komplex sind und nicht mit einer einfachen Erklärung verstanden werden können. Das Bewusstsein des Menschen an sich zu verstehen, ist bereits ein außerordentlich komplexes Unterfangen und seit vielen Jahren Gegenstand diffiziler naturwissenschaftlicher, theologischer und philosophischer Abhandlungen. So ist es nicht verwunderlich, dass Narkose - frei übersetzt das Erstarren des Bewusstseins (griechisch: nárkōsis - „Erstarrung“) - ebenfalls für viele schwer verständlich ist.

Gerade der vom Patienten akzeptierte Verlust an Bewusstsein setzt somit ein enormes Vertrauen in den Anästhesiologen voraus. Vertrauen in seine Kompetenz, sein Wissen und eben darin, dass er Komplikationen antizipiert und gar nicht erst entstehen lässt, dass er während der Narkose und danach auf den Patienten und seine Vitalfunktionen lückenlos Acht gibt und alles Erdenkliche unternimmt, dass die Narkose sicher ist.

\section{Aber wie sicher sind nun unsere Narkosen?}

Was können wir den Patienten hierüber aufrichtig sagen, damit sie sich eben keine $z u$ großen, damit unberechtigten, Sorgen machen und wir ein angemessenes Gefühl der Sicherheit vermitteln?

Hier geben Boehm et al. ausgesprochen interessante Antworten, indem sie sehr übersichtlich und dezidiert die entsprechende Datenlage darstellen und bewerten. Die anästhesieassoziierte Sterblichkeit liegt seit vielen Jahren mit 0,68-0,82/100.000 chirurgische Fälle auf einem sehr niedrigen Niveau. Dies sind beruhigende Zahlen, bedenkt man, wie existenziell „Anästhesie“ für die Patienten sein kann. Eine Ursache hierfür ist sicherlich, dass der Anästhesist mittlerweile über viele Kontroll- und Steuerungsmöglichkeiten verfügt. Er kann die Funktion des ,inneren Autopiloten “ grundsätzlich weit mehr kontrollieren und beeinflussen als der Patient selbst. Der Anästhesiologe überwacht kontinuierlich Blutdruck, Herzfrequenz, Sauerstoffsättigung, Kohlenstoffdioxid $\left(\mathrm{CO}_{2}\right)$ etc., und er nimmt direkt Einfluss auf diese Parameter - was auch Ängste auslöst. Am eindrucksvollsten ist sicherlich die Übernahme der Atmung durch eine kontrollierte maschinelle Beatmung während der Allgemeinanästhesie. Der Anästhesist kann zudem sofort, zielgerichtet und höchst effektiv medikamentös entgegenwirken, wenn der Blutdruck nach oben oder unten entgleist, unmittelbar eine zu langsame oder zu schnelle Herzfrequenz medikamentös oder apparativ (Schrittmacher/Defibrillator) korrigieren, einen plötzlichen Herztod durch eine sofortige 
Reanimation verhindern (zugegeben sicherlich nicht immer, aber weit besser als der Patient selbst). Deshalb ist Anästhesie heutzutage sehr sicher.

Allerdings, Sicherheit ist relativ und Statistiken je nach Blickwinkel verschieden, hierzu im Folgenden ein einfaches Beispiel.

\section{Vergleich mit der Fliegerei hinkt}

Anästhesie wird gerne mit der Fliegerei verglichen. Bei beiden sind "Start“ und „Landung“ mit dem größten Risiko verbunden, hat man mal eine gewisse „Flughöhe" erreicht, so ist bei beiden Vorgängen die stabilste und damit auch sicherste Phase eingetreten. Global betrachtet, ist das Flugzeug unangefochten das sicherste Verkehrsmittel. Die Betrachtungsweise ist für die Frage nach der Sicherheit aber entscheidend. Vergleicht man z. B. 3 verschiedene Verkehrsmittel, so beträgt in der EU für das Flugzeug und den Zug die Zahl der Toten/1 Mio. Reisekilometer 0,035, das Auto liegt hier mit 0,7 unrühmlich weit vorne (Faktor 20). Betrachtet man jedoch die Toten/1 Mio. Reisestunden ergibt sich ein anderes Bild: Auf das Flugzeug kommen 16 Tote, auf den Zug hingegen nur 2. Das Auto kann hier immer noch nicht punkten, bei dieser Ansicht sind es ganze 25 und damit immer noch die meisten Toten [1]. So gesehen, sitzt man also im Zug 8-mal sicherer als im Flugzeug, allerdings braucht man deutlich länger, um die gleiche Distanz zu überwinden, was das Risiko wieder erhöht. Setzte man jedoch die Zahl der Toten mit der Zahl der transportierten Personen ins Verhältnis, so schnitt das Flugzeug sogar weit schlechter ab als das Auto [4]. Die Sicherheit der Anästhesie wird aber üblicherweise genau so berechnet, nämlich nach der Zahl der Fälle bzw. Narkosen. Eine Betrachtung nach Kilometern wäre natürlich Unfug, ausgenommen womöglich Transporte in Narkose, selten misst man jedoch die Distanzen. Die Betrachtung nach Anästhesiestunden wäre aber äußerst interessant. Damit könnte man z. B. die anästhesieassoziierte Letalität/1 Mio. Stunden mit der natürlichen Sterblichkeit/1 Mio. Stunden (also der Normalbevölkerung ohne Narkose; Stichwort plötzlicher Herztod) relativieren/ korrigieren, um die tatsächlich anästhesiebedingte Letalität weiter zu entschlüsseln.

Grundsätzlich kann man Verkehrsmittel nur sehr eingeschränkt mit der Anästhesie/Narkose vergleichen: Reisen unternimmt man ja nicht nur, weil man muss, sie haben auch Freizeitcharakter, den Narkosen wohl nie erlangen werden können. Bei Reisen mit dem Flugzeug, Zug oder Auto gilt: „no risk no fun“. Narkosen machen den Patienten in der Regel eher wenig Spaß, sie erfüllen auch meist keinen Selbstzweck. Im Flugzeug mag es den ein oder anderen beruhigen, dass der Pilot mitfliegt. Der Anästhesist hingegen stirbt nie mit, wenn er einen Fehler macht. Zudem ist der Patient nicht einfach als Passagier zu betrachten. Er erträgt während der Narkose meist zusätzlich ein im wahrsten Sinne des Wortes einschneidendes Ereignis durch den Operateur. Anästhesie lässt sich vom chirurgischen Eingriff naturgemäß schwer trennen, das Flugzeug hingegen wird während des Fluges eher selten repariert. Auch gibt es kaum „Notfallflüge" mit nichtintakten, nichtgewarteten, voll besetzten Passagiermaschinen, in der Anästhesie hingegen gehören Notfallszenarien, zumindest in Kliniken der Maximalversorgung, zum Alltag.

Boehm et al. legen auch den Finger in eine offene Wunde, denn, insgesamt betrachtet, ist die durchschnittliche postoperative Letalität mit 0,8-4\% deutlich zu hoch. So lässt die dargelegte Analyse bezüglich „failure to rescue“ vermuten, dass allein in Deutschland jährlich 60.000 Todesfälle vermeidbar wären. Rückgrat zeigt man nicht, indem man sich wegdreht; dieser Zahl müssen wir Ärzte uns zukünftig stellen! Die Lösungsansätze und Verbesserungen, die Boehm et al. in ihrem Impuls gebenden Beitrag aufzeigen, implizieren gerätetechnische, organisatorische bis hin $\mathrm{zu}$ strukturellen Veränderungen. Geräte und Technik allein (z. B. „smart wearables“) sind sicherlich nicht die Lösung. So ist z. B. in den 1980er Jahren nach Einführung des Antiblockiersystems (ABS) zunächst die Zahl der Unfälle gerade mit den Autos, die mit ABS ausgerüstet waren, erheblich angestiegen, da man u. a. glaubte, der Bremsweg würde dadurch drastisch reduziert werden und man könne deut- lich schneller fahren - ein Trugschluss $[2,3]$. Ähnliches sah man bei Einführung des Sicherheitsgurts. Sicherheits-Tools können also ein zu starkes Gefühl der Sicherheit erzeugen und damit die gewünschte Risikoreduktion verhindern [3]. Dieses als „Sicherheitskompensation“ oder „Peltzman-Effekt“ [2,5] benannte Phänomen betrifft auch die Medizin und die Anästhesie: Trotz Einführung neuer, moderner Überwachungsmöglichkeiten konnte man $\mathrm{z}$. B. das sehr geringe Risiko, an einer Narkose zu versterben, in den letzten Jahrzehnten nicht weiter senken. Sicherlich da zunehmend große, risikoreiche Operationen bei immer kränker werdenden Patienten durchgeführt wurden. Dies ist aber keineswegs nur negativ zu sehen. Immerhin können mittlerweile Operationen mit zwar hohem, aber heute vertretbarem Risiko durchgeführt werden, die vor Jahrzehnten noch nicht möglich waren.

Das hohe Risiko in der postoperativen Phase müssen wir jedoch definitiv reduzieren, hier dürfen die anzustrebenden Verbesserungen nicht zu einer Risikoerhöhung verleiten.

Die von Boehm et al. behandelte Thematik ist also sehr komplex, sie reicht weit über die Anästhesie hinaus und wird sicherlich das perioperative Management in Zukunft prägen. Neues Wissen zielgerichtet $\mathrm{zu}$ implementieren und notwendige Verbesserungen konsequent auch interdisziplinär - umzusetzen, dies sind die notwendigen Schritte, um die perioperative und insbesondere die postoperative Phase noch sicherer zu gestalten. Erst dann schafft Wissen größere Sicherheit.

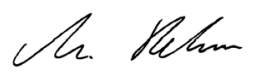

M. Rehm

\section{Korrespondenzadresse}

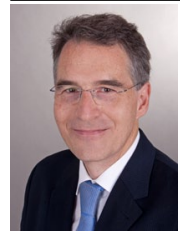

Prof. Dr. M. Rehm

Klinik für Anaesthesiologie, Klinikum der Universität München, Campus Großhadern, Marchioninistr. 15, 81377 München markus.rehm@ med.uni-muenchen.de 
Danksagung. Der Autor bedankt sich bei Julian Rehm und Dr. Christian Kowalski für die vorzügliche Korrektur des Manuskripts!

Interessenkonflikt. M. Rehm gibt an, dass kein Interessenkonflikt besteht.

\section{Literatur}

1. Bernau P (2015) Die Gefahren des Fliegens. Frankfurter Allgemeine. http://www.faz.net/aktuell/gesellschaft/absturz-in-den-alpen/wie-gefaehrlichist-fliegen-die-risiko-statistiken-13505192.html

2. Biehl B, Aschenbrenner M, Wurm G (1987) Einfluß der Risikokompensation auf die Wirkung von Verkehrssicherheitsmaßnahmen am Beispiel ABS. Unfall Sicherheitsforschung Straßenverkehr 63:65-70

3. Blawat K (2007) Risiko statt Sicherheit: Der Mensch sucht die Gefahr. Spiegel online - Wissenschaft. http://www.spiegel.de/wissenschaft/mensch/ risiko-statt-sicherheit-der-mensch-sucht-die-gefahr-a-505448.html

4. Stauber R (2000) Unfallstatistik, das Verwirrspiel um die Flugsicherheit. Beobachter 5/2000. http:// www.beobachter.ch/konsum/reisen/artikel/unfallstatistik-das-verwirrspiel-um-die-flugsicherheit/

5. Traynor TL (1993) The Peltzman hypothesis revisited: an isolated evaluation of offsetting driver behaviour. J Risk Uncertainty 7:237-247

\section{Bundesweites Berichts- \& Lernsystem CIRS-AINS bietet Mehrwert}

Seit April 2010 wurden über 4.000 Berichte in dem bundesweit verbreiteten Critical Incident Reporting System der Anästhesisten, CIRSAINS, veröffentlicht. Ziel der von dem Berufsverband der Anästhesisten e. V. (BDA) und der Deutschen Gesellschaft für Anästhesiologie \& Intensivmedizin e. V. (DGAI) betriebenen Datenbank ist eine aktive und nachhaltige Erhöhung der Patientensicherheit durch Sammlung, Auswertung und Weitergabe von Informationen über Ereignisse, Zwischenfälle und Komplikationen mit und ohne Patientenschaden vor, während und nach der Anästhesie. Doch geht das Angebot von CIRS-AINS weit über übliche Standards von medizinischen Incident-Reporting-Systemen hinaus. Bislang nehmen 97 Kliniken an CIRSAINS teil, davon 13 Universitätskliniken. „Monatlich werden durchschnittlich 50 Fälle gemeldet. Von den jährlich knapp 600 gemeldeten Fällen wurden 345 Ereignisse im Jahr 2014 durch das Expertenteam BDA/DGA analysiert.

Was aus einem Fallbericht werden kann, zeigt anschaulich der Bericht einer Pflegekraft. Hier gelang es bei einer geplanten Operation nicht, die Narkose und Intubation mit Larynxmaske in Bauchlage des Patienten einzuleiten. Auch auf eine Präoxigenierung wurde verzichtet. Obwohl die Intubation in Bauchlage keine in Lehrbüchern erwähnte und valide Methode ist, ist diese Vorgehensweise gängige Praxis, wie nach der Meldung des Falles festgestellt wurde. So wird das Umlagern umgangen und wertvolle Zeit gespart. Als Ergebnis des Berichtes wurde festgehalten, dass bei der Verwendung der Larynxmaske in Bauchlage vorab Nutzen und Risiken sorgfältig abgewogen werden müssen. Gerade bei Patienten mit pulmonalen Risiken kann der "Goldstandard" Intubation von Vorteil sein. Außerdem erfolgte der dringende Rat, Patienten vor der Narkoseeinleitung grundsätzlich zu präoxygenieren. Dieser Fall wurde nicht nur im Meldesystem CIRS-AINS behandelt. Es folgte eine Umfrage unter den im vertragsärztlichen ambulanten Bereich tätigen Mitgliedern von BDA und DGAl, um Informationen zu Art und Umfang des Ein- satzes dieser Narkoseform zu generieren. Die Umfrageergebnisse wurden in der Fachzeitschrift Anästhesiologie \& Intensivmedizin gemeinsam mit der Einschätzung publiziert, dass diese Methode aktuell nicht als sicher empfohlen werden kann. Über das reine Meldesystem „Berichten \& Lernen“ hinaus, wurde so nicht nur der Einzelfall aufgearbeitet, sondern einer Vielzahl von Anästhesisten ein wichtiger Erfahrungswert weitergegeben.

Ähnlich verhielt es sich bei Berichten über Partikelkontaminationen nach dem Aufziehen von Arzneimitteln. Die Häufung eingehender Fallberichte führte dazu, dass der Ursache und einer Lösung intensiv nachgegangen wurde. Obwohl schon in der Vergangenheit der Nutzen von Filtersystemen für dieses Problem belegt war, fand deren Verwendung keinen Einzug in die Praxis. Studien hierzu und die Veröffentlichung der Ergebnisse sind nun die Basis für eine Überarbeitung der Fachinformation für Infusionslösungen, die in Planung ist.

Info-Tipp:

Auf der Webseite https://www.cirs-ains.de/ cirs-ains.html haben auch nicht registrierter Nutzer die Möglichkeit, über den Button „Berichten und Lernen“ Fallberichte einzugeben und zu lesen. 